\title{
Feasibility of MRI based oxygenation imaging for the assessment of acute limb ischemia
}

\author{
Bihui Zhang ${ }^{1 \#}$, Chengyan Wang ${ }^{2,3 \#}$, Haochen Wang ${ }^{1}$, Hanjing Kong ${ }^{3}$, Fei Gao ${ }^{4}$ Min Yang ${ }^{1}$, Jue Zhang $^{3,4}$ \\ ${ }^{1}$ Department of Interventional Radiology and Vascular Surgery, Peking University First Hospital, Beijing 100034, China; ${ }^{2}$ Human Phenome \\ Institute, Fudan University, Shanghai 201203, China; ${ }^{3}$ Academy for Advanced Interdisciplinary Studies, Peking University, Beijing 100871, China; \\ ${ }^{4}$ College of Engineering, Peking University, Beijing 100871, China \\ Contributions: (I) Conception and design: M Yang, J Zhang; (II) Administrative support: M Yang, J Zhang; (III) Provision of study materials or \\ patients: B Zhang, C Wang; (IV) Collection and assembly of data: B Zhang, C Wang, H Wang; (V) Data analysis and interpretation: B Zhang, C \\ Wang, H Kong, F Gao; (VI) Manuscript writing: All authors; (VII) Final approval of manuscript: All authors. \\ \#These authors contributed equally to this work. \\ Correspondence to: Min Yang, MD. Department of Interventional Radiology and Vascular Surgery, Peking University First Hospital, No. 8 Xishiku \\ Street, Xicheng District, Beijing 100034, China. Email: dryangmin@gmail.com; Jue Zhang, PhD. Academy for Advanced Interdisciplinary Studies, \\ Peking University, No. 5 Summer Palace Road, Haidian District, Beijing 100871, China. Email: zhangjue@pku.edu.cn.
}

\begin{abstract}
Background: Acute limb ischemia (ALI) can lead to death and amputation. Evaluating the severity of ischemia is important but difficult, through current methods of examination. The purpose of this research was to demonstrate the feasibility of magnetic resonance imaging (MRI) susceptibility-based imaging techniques for use in assessing muscle oxygenation alterations in ALI.

Methods: ALI animal models were established in 11 rabbits. Their left iliac arteries were embolized by microspheres. MRI scans were conducted 24 hours before (Pre) and 1 hour (Post 1) and 3 hours (Post 2) after the procedure. A susceptibility model was used to calculate skeletal muscle oxygenation extraction fraction (SMOEF) and relaxation rate (R2'). T2 weighted (T2w) imaging and diffusion-weighted (DW) imaging were performed.

Results: The average calf muscle SMOEF in the embolized hindlimbs increased from $0.43 \pm 0.02$ (Pre) to $0.48 \pm 0.02$ (Post 1 ) and $0.50 \pm 0.02$ (Post 2), both $\mathrm{P}<0.05$. The R2' value increased from $13.01 \pm 2.31 \mathrm{~s}^{-1}$ (Pre) to $16.78 \pm 2.28 \mathrm{~s}^{-1}$ (Post 1 ) and $17.90 \pm 3.29 \mathrm{~s}^{-1}$ (Post 2), both $\mathrm{P}<0.05$. No significant changes of SMOEF and R2' were found after embolization in the contralateral hindlimbs. Apparent diffusion coefficient (ADC) values derived from DW imaging remained unchanged at different stages compared to before the procedures (all $\mathrm{P}>0.05)$. No abnormal signals were observed in the anatomical T2 $\mathrm{w}$ images at Post 1 and Post 2.

Conclusions: This study demonstrates the feasibility of using SMOEF for the assessment of oxygenation alterations in ALI models. SMOEF is more sensitive than T2w and DW imaging in detecting acute muscle ischemia at an early stage.
\end{abstract}

Keywords: Ischemia; magnetic resonance imaging (MRI); peripheral vascular diseases

Submitted Dec 03, 2019. Accepted for publication Feb 07, 2020.

doi: $10.21037 /$ atm.2020.02.139

View this article at: http://dx.doi.org/10.21037/atm.2020.02.139

\section{Introduction}

Acute limb ischemia (ALI) is an emergent condition caused by an abrupt decrease or interruption in the blood flow supply to a limb (1). The incidence of ALI is reported to be about 150 per million people per year (2). Mortality rates range from $15-20 \%$, the major amputation rate is up to $25 \%$, and renal insufficiency is up to $20 \%$ (3). The prognosis is determined by the duration and severity of ischemia and hypoxia $(3,4)$. Early intervention, either endovascular or surgical therapy, to the threatened limb, is 
crucial for prognosis (5). Thus, urgent recognition of ALI and the evaluation of limb viability are required to reduce death and complications.

To date, imaging modalities, including duplex ultrasound, computed tomography angiography, magnetic resonance angiography, and invasive angiogram, are the most widely used means of assessing ALI (5). However, these methods can only reflect the blood flow condition, rather than the severity of tissue oxygenation. Because oxygen supply and demand are varied in every patient, an assessment of blood flow may not provide physiological insight into the extent and severity of ischemia (6). Since muscle oxygenation is sensitive to tissue ischemia, measuring muscle tissue oxygenation can be an effective way of assessing lower limb ischemic disease $(5,7)$. In the past decade, near-infrared spectroscopy (NIRS) has been used to monitor muscle oxygen saturation (8). However, the maximum penetration depth of NIRS is only two centimeters, which cannot be used to evaluate the whole muscle. In addition, skin pigmentation and adipose tissue were found to influence NIRS measurements significantly $(9,10)$. Therefore, a novel method of measuring muscle tissue ischemia is needed for evaluating limb viability in ALI.

The Magnetic Resonance Imaging (MRI)-based blood oxygenation level-dependent (BOLD) technique has been successfully applied for individuals suffering from peripheral arterial diseases (9). BOLD contrast is based on the characteristics of magnetic susceptibility of oxyhemoglobin and deoxyhemoglobin. Conventional BOLD imaging is not applicable for evaluating ischemia severities because of its qualitative characteristics. Recently, a susceptibility model-based MRI technique has been proposed for the quantification of skeletal muscle oxygen extraction fraction (SMOEF) (10), which seems to be an effective approach for evaluating muscle ischemia in vivo $(11,12)$.

The purpose of this research was to assess the feasibility of a noninvasive MRI-based oxygenation imaging for use in evaluating SMOEF in ALI animal models.

\section{Methods}

\section{ALI animal models}

The ALI models in this study were established in $11 \mathrm{New}$ Zealand white rabbits (weight range, $2.8-3.5 \mathrm{~kg}$ ). The experimental protocols and all embolization procedures were scrutinized by and received approval from the local Institutional Animal Care and Use Committee (J201740).
Vascular specialists conducted the procedures under the guidance of an interventional image-guided system (INNOVA 4100, GE Healthcare, Waukesha, USA). Before the procedure, rabbits were placed in the supine position on the operating table with a mask closely fitted to cover the whole mouth, and a calibrated vaporizer was used to deliver ventilation with isoflurane (2-3\%). A flexible tube supplied air to the mask, with a flow rate of $2.5 \mathrm{~L} / \mathrm{min}$. During the embolization procedure, the left carotid artery was dissected before the placement of a 4 French sheath. Then, a 4 French vertebral catheter was introduced and manipulated, so the left common iliac artery could be reached under the guidance of fluoroscopy. The embolization was then performed by injecting a suspension of microspheres $(2.0 \mathrm{mg}, 100-$ $150 \mu \mathrm{m}$, Hepasphere, Merit Medical, Rockland, MA) into the left iliac artery slowly to avoid backflow. The endpoint of embolization is the stagnation of blood flow in the ipsilateral iliac artery. Hindlimb digital subtraction angiographies (DSA, flow velocity $=1.5 \mathrm{~mL} / \mathrm{s}$, flow time $=3 \mathrm{~s}$ ) were carried out prior to and following the embolization in each of the rabbits to confirm that embolization was successful.

The rabbits were transferred for MRI scans immediately after the procedure. The experimental protocol is set out in Figure 1. MRI scans were conducted 24 hours before the embolization procedure (Pre) and 1 hour (Post 1) and 3 hours (Post 2) after the procedure.

\section{MRI scans}

A 3.0 Tesla clinical MRI scanner (Signa Excite ${ }^{\mathrm{TM}}$; GE Medical System, Milwaukee, Wisconsin, USA) was used to carry out each MRI scan. Body coil and an 8-channel knee coil were used for signal transmission and reception, respectively. The rabbits were fixed in a knee coil with foamed plastics wrapped around the body to make sure both legs could be imaged without repositioning.

T2-weighted (T2w) imaging covering both legs were obtained using a fast spin-echo (FSE) sequence with field of view $(\mathrm{FOV})=150 \times 150 \mathrm{~mm}^{2}$, repetition time $(\mathrm{TR})=3,000 \mathrm{~ms}$, echo time $(\mathrm{TE})=80 \mathrm{~ms}$, acquired matrix $=256 \times 256$, and slice thickness $=6 \mathrm{~mm}$. To assess the lower extremity oxygenation level, a multi-echo gradient and spin echo (MEGSE) sequence were applied in the coronal plane to acquire mixed $\mathrm{T} 2$ and $\mathrm{T} 2{ }^{*}$-weighted images. A total of 32 echoes with an echo spacing of $3.7 \mathrm{~ms}$ were applied in the MEGSE sequence. The spin echo occurred at the seventh echo, as described previously (13). The detailed imaging parameters of MEGSE were: FOV $=150 \times 150 \mathrm{~mm}^{2}$, TR $=1,500 \mathrm{~ms}$, TE 


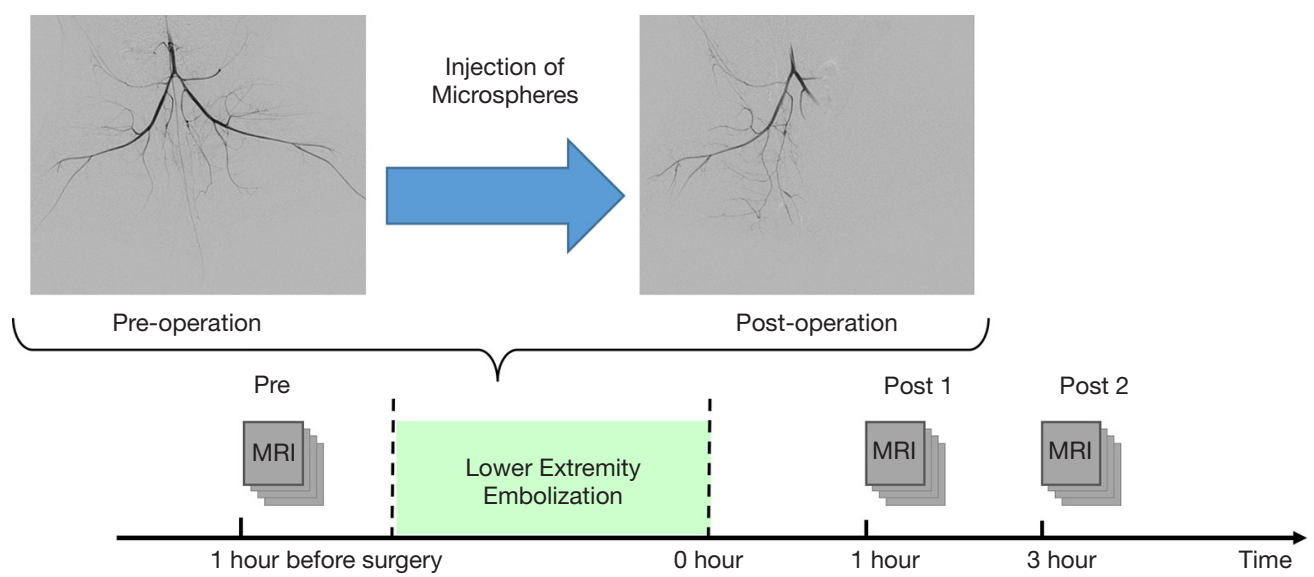

Figure 1 Diagram of the study protocol of the experiment. The three time points for MR imaging are defined as follows: Pre: baseline (before femoral artery embolization operation); Post 1: $1 \mathrm{~h}$ after unilateral artery embolization operation; Post 2: $2 \mathrm{~h}$ after unilateral artery embolization operation.

$=56 \mathrm{~ms}$, acquired matrix $=128 \times 128$, slice thickness $=6 \mathrm{~mm}$. Diffusion-weighted (DW) images were immediately acquired after MEGSE scan with identical geometrical parameters as MEGSE. $b$ values of 0 and $800 \mathrm{~s} / \mathrm{mm}^{2}$ were applied for the DW imaging. The total scan time for the three sequences was approximately 10 minutes.

\section{Data analysis}

Data analysis was carried out with the aid of a home-built Matlab (MathWorks Inc., Natick, MA, USA) program. Raw images acquired from the MRI scanner were reviewed by an experienced radiologist (with 7 years of experience) to avoid artifacts from motion or other sources. A Gaussian low-pass filter (kernel size $=3 \times 3$ ) was used on every MEGSE image to enhance the signal-to-noise ratio before further analysis.

The calculation of skeletal muscle oxygenation extraction fraction (SMOEF) was performed according to a susceptibility model using the magnetic properties of oxyhemoglobin/deoxyhemoglobins (10). Briefly speaking, the MEGSE signal can be written as:

$S_{L}(\tau)=\rho(1-\lambda) \cdot \exp (-\lambda \cdot \delta \omega \cdot 2 \tau+\lambda) \cdot \exp \left(-\frac{T E}{T 2}\right) \cdot\left(1-\exp \left(-\frac{T R}{T 1}\right)\right)[1]$

for $\delta \omega \cdot 2|\tau|>1.5$, and:

$S_{S}(\tau)=\rho(1-\lambda) \cdot \exp \left(-0.3 \lambda \cdot(\delta \omega \cdot 2 \tau)^{2}\right) \cdot \exp \left(-\frac{T E}{T 2}\right) \cdot\left(1-\exp \left(-\frac{\mathrm{TR}}{\mathrm{T} 1}\right)\right)[2]$

for $\delta \omega \cdot 2|\tau|<1.5$, in which $\rho$ is the spin density, $\lambda$ is the volume fraction of muscle occupied by the susceptibility sources, $\delta \omega$ is the characteristic frequency shift defined as: $\delta \omega=\frac{4}{3} \pi \cdot \gamma \cdot \Delta \chi_{0} \cdot H c t \cdot B_{0} \cdot O E F$

where $\gamma$ is the gyromagnetic ratio, $\Delta \chi_{0}$ is the susceptibility difference between fully oxygenated and fully deoxygenated blood, Hct is the fractional hematocrit, and OEF is the muscle oxygenation level. A nonlinear least-squares curve fitting function was used to fit this model on a pixel-bypixel basis. In addition, the relaxation rate (R2') is obtained as a semi-quantitative depiction of tissue ischemia, which is defined as:

$R 2^{\prime}=\lambda \cdot \delta \omega$

\section{Statistical analysis}

All statistical analyses were conducted using Origin software (version 10.2). Regions of interest (ROIs) were selected on the calf muscle in T2w images. The ROIs were selected to avoid signals from bones and vessels by an experienced radiologist (with 7-years experience). Relative SMOEF/ $\mathrm{R} 2$ '/apparent diffusion coefficient (ADC) was defined as the ratio of SMOEF/R2'/ADC on the ischemic limb to the contralateral limb. All the results are reported as mean \pm standard deviation. Differences between groups were analyzed using the paired two-sided Student $t$-test. $\mathrm{P}<0.05$ suggested statistically significant difference.

\section{Results}

One rabbit died during embolization. DSA and MRI data 

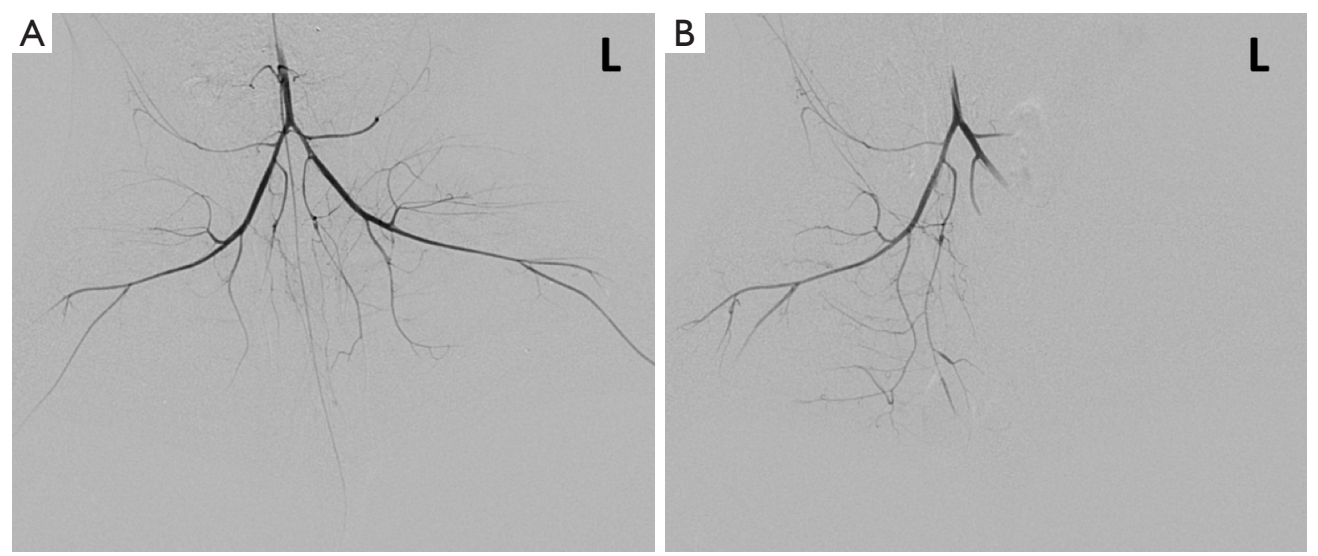

Figure 2 DSA of the hindlimb arteries before (A) and after (B) embolization. Arteries of the left hindlimb are occluded and stagnation of contrast medium in the left iliac artery is shown in the DSA image after the embolization procedure. DSA, digital subtraction angiography.

were successfully obtained from each of the remaining rabbits $(\mathrm{N}=10)$. The quality of the images was adequate for quantitative analysis. The stagnation of the contrast medium in the left iliac artery was observed in the DSA image in all the animal models. A representative case of embolization is shown in Figure 2. The corresponding anatomical T2w images, R2' maps, and SMOEF maps before (Pre) and after the procedure (Post 1 and Post 2) are displayed in Figure 3. The muscle R2' and SMOEF of the ischemic leg rose obviously after the procedure (Figure 3, red arrow). On the contrary, abnormal signals were not observed in the $\mathrm{T} 2 \mathrm{w}$ image at both Post 1 and Post 2 (Figure 3). Increased signal intensity was observed in the DW images $\left(b=800 \mathrm{~s} / \mathrm{mm}^{2}\right)$ at Post 2, but the change was not obvious at Post 1 (Figure 4).

There was a significant increase in the value of SMOEF of the ischemic hindlimb from $0.43 \pm 0.02$ (Pre) to $0.48 \pm 0.02$ (Post $1, \mathrm{P}<0.05$ ), and to $0.50 \pm 0.02$ (Post $2, \mathrm{P}<0.05$ ) on average. In contrast, no differences of SMOEFs were found in the contralateral hindlimb at different stages (Pre: $0.42 \pm 0.01$; Post $1: 0.41 \pm 0.02, \mathrm{P}=0.87$; Post 2 : $0.42 \pm 0.02$, $\mathrm{P}=0.29$ ) (Figure 5A). Correspondingly, calf muscle R2' increased significantly from $13.01 \pm 2.31 \mathrm{~s}^{-1}$ (Pre) to $16.78 \pm 2.28 \mathrm{~s}^{-1}$ (Post $1, \mathrm{P}<0.05$ ), and to $17.90 \pm 3.29 \mathrm{~s}^{-1}$ (Post 2, $\mathrm{P}<0.05)$. No differences in calf muscle R2' were found in the contralateral leg at different stages (Pre: $12.62 \pm 1.76 \mathrm{~s}^{-1}$; Post 1: $12.88 \pm 1.02 \mathrm{~s}^{-1}, \mathrm{P}=0.69$; Post $2: 12.84 \pm 2.43 \mathrm{~s}^{-1}, \mathrm{P}=0.82$ ) (Figure $5 B$ ). There was no change in the ADC values of the ischemic calf muscle after the embolization procedure (Pre: $1.50 \times 10^{-3} \pm 0.12 \times 10^{-3} \mathrm{~mm}^{2} / \mathrm{s}$; Post $1: 1.52 \times 10^{-3} \pm 0.08 \times$ $10^{-3} \mathrm{~mm}^{2} / \mathrm{s}, \mathrm{P}=0.60$; Post $2: 1.51 \times 10^{-3} \pm 0.09 \times 10^{-3} \mathrm{~mm}^{2} / \mathrm{s}$, $\mathrm{P}=0.76$ ) (Figure 5C). In the ischemic limb, calf muscle SMOEFs were found to be different between Post 1 and
Post $2(\mathrm{P}<0.01)$, but calf muscle $\mathrm{R} 2$ 's were not $(\mathrm{P}=0.39)$.

The relative SMOEF increased from $1.03 \pm 0.08$ (Pre) to $1.15 \pm 0.06$ (Post $1, \mathrm{P}<0.05$ ) and $1.18 \pm 0.09$ (Post 2, $\mathrm{P}<0.05$ ), and the relative R2' increased significantly from $1.04 \pm 0.15$ (Pre) to $1.31 \pm 0.21$ (Post $1, \mathrm{P}<0.05$ ) and $1.45 \pm 0.45$ (Post 2, $\mathrm{P}<0.05$ ) (Figure 6). The relative $\mathrm{ADC}$ values were similar at different stages (Post $1 v s$. Pre, $\mathrm{P}=0.33$; Post $2 v s$. Pre, $\mathrm{P}=0.82$ ).

\section{Discussion}

In our study, we examined the feasibility of using a magnetic resonance susceptibility-based imaging technique for the quantitative measurement of muscle oxygenation alterations in ALI animal models. The SMOEF calculated from the MRI MEGSE sequence is more sensitive than conventional anatomical T2 $\mathrm{w}$ and DW images in detecting muscle oxygenation alterations at an early stage of ALI. The SMOEF has great potential to be an effective biomarker for assessing the severity of ALI.

In recent decades, emerging MRI techniques have been developed to evaluate muscle ischemia. BOLD imaging has been widely used to evaluate muscle ischemia in patients with peripheral arterial diseases (9). However, conventional BOLD imaging only provides a qualitative assessment of the tissue pressure of oxygen $\left(\mathrm{pO}_{2}\right)$, which cannot be used for the longitudinal evaluation of the same individual or for comparisons among different individuals. The SMOEF measurement based on a susceptibility model has been developed for the quantification assessment of tissue ischemia (10). This technique has been applied in the evaluation of oxygenation of the brain (14), the kidneys (15), 


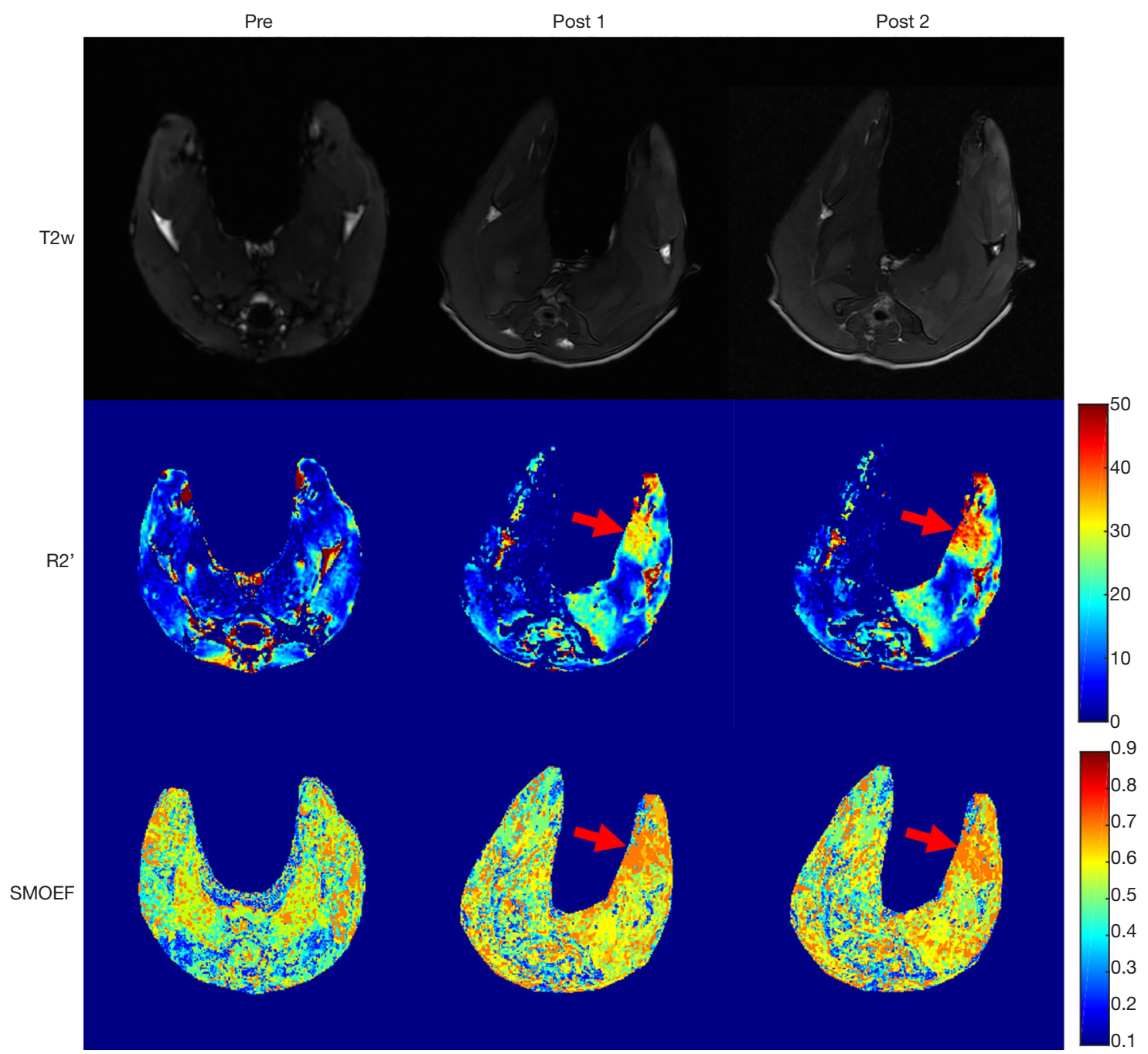

Figure 3 Representative anatomical T2w, R2' and SMOEF maps of ischemic muscles after left iliac artery embolization. It is observed that R2' and SMOEF increase obviously in the calf muscle of ischemic hindlimb (red arrow). No abnormal signal is found by T2w. T2w, T2 weighted image; SMOEF, skeletal muscle oxygenation extraction fraction.

and the muscles $(11,12)$. Our study also proved that SMOEF could be used to detect pathological limb ischemia effectively.

In our experiment, we observed an $11.6 \%$ elevation of calf muscle oxygenation level within 1 hour following the embolization procedure (Post 1), and 16.3\% elevation within 3 hours after the procedure (Post 2). Correspondingly, the calf muscle R2' showed an increase of $29.1 \%$ at Post 1 and $37.7 \%$ at Post 2. A larger variation was observed in the calf muscle R2' compared to SMOEF, because of the mixed sources of imaging contrast, including magnetic field $\mathrm{B}_{0}$ inhomogeneity, blood volume, blood flow, and tissue oxygenation level. The ischemic status of calf muscle was not detected by both $\mathrm{T} 2 \mathrm{w}$ and $\mathrm{DW}$ images within 1 hour. Tissue inflammation was observed in DW images $\left(b=800 \mathrm{~s} / \mathrm{mm}^{2}\right)$ within 3 hours after the procedure. This can be explained by the fact that once injected into the blood, the microspheres deposit in small arteries of similar diameter $(100-150 \mu \mathrm{m})$. The interruption of blood flow immediately impedes perfusion and promotes the accumulation of deoxyhemoglobin, leading to an increase of SMOEF and R2'. On the other hand, inflammation develops gradually in endothelial cells, which are then detected by DW imaging.

In this study, ALI animal models were established by trans-arterial embolization, which is different from 


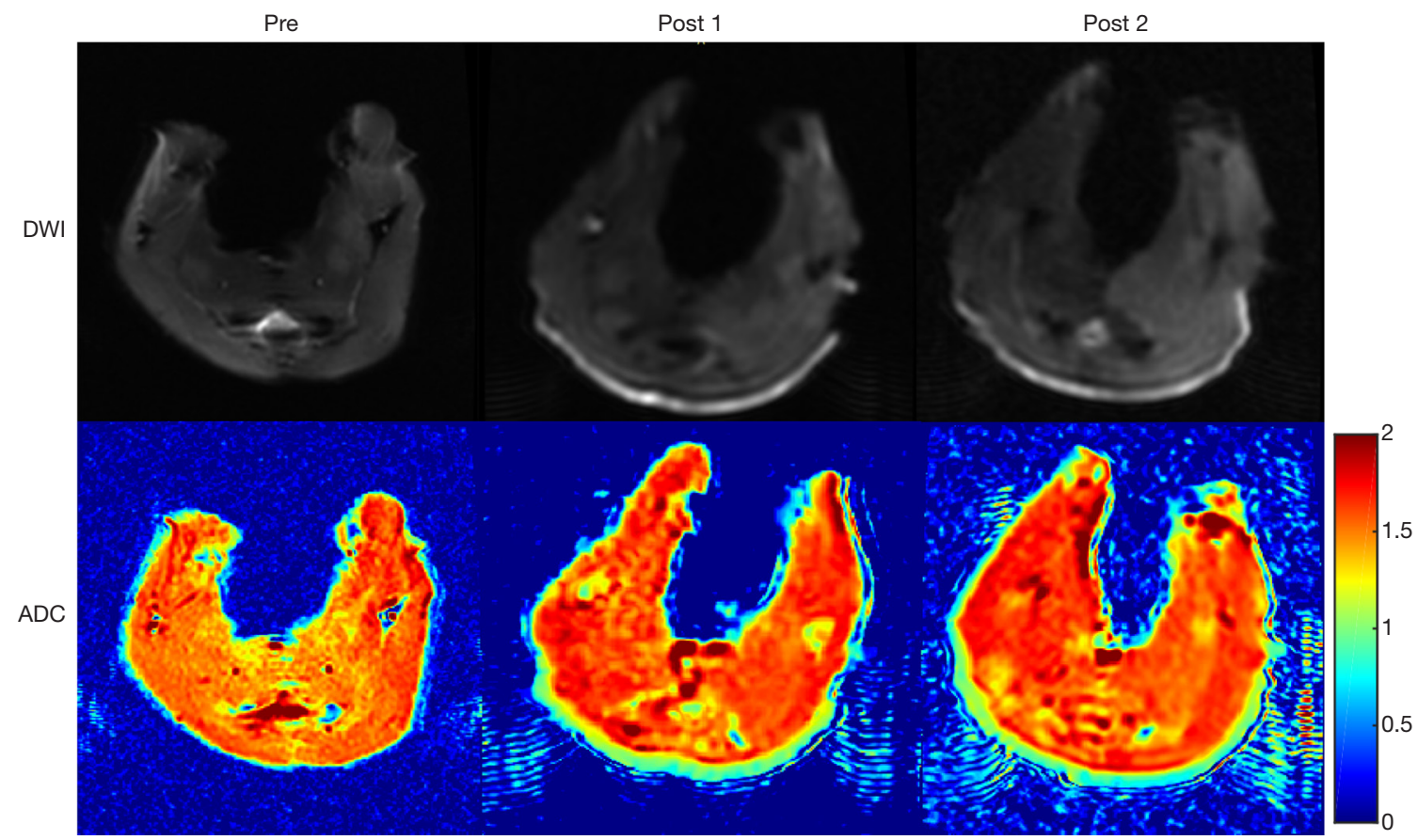

Figure 4 Representative DWI and ADC maps of ischemic muscles after left iliac artery embolization. Increased signal intensity is observed in the DW images $\left(\mathrm{b}=800 \mathrm{~s} / \mathrm{mm}^{2}\right)$ at Post 2, but the change is not obvious at Post 1 . DWI, diffusion weighted imaging; ADC, apparent diffusion coefficient.
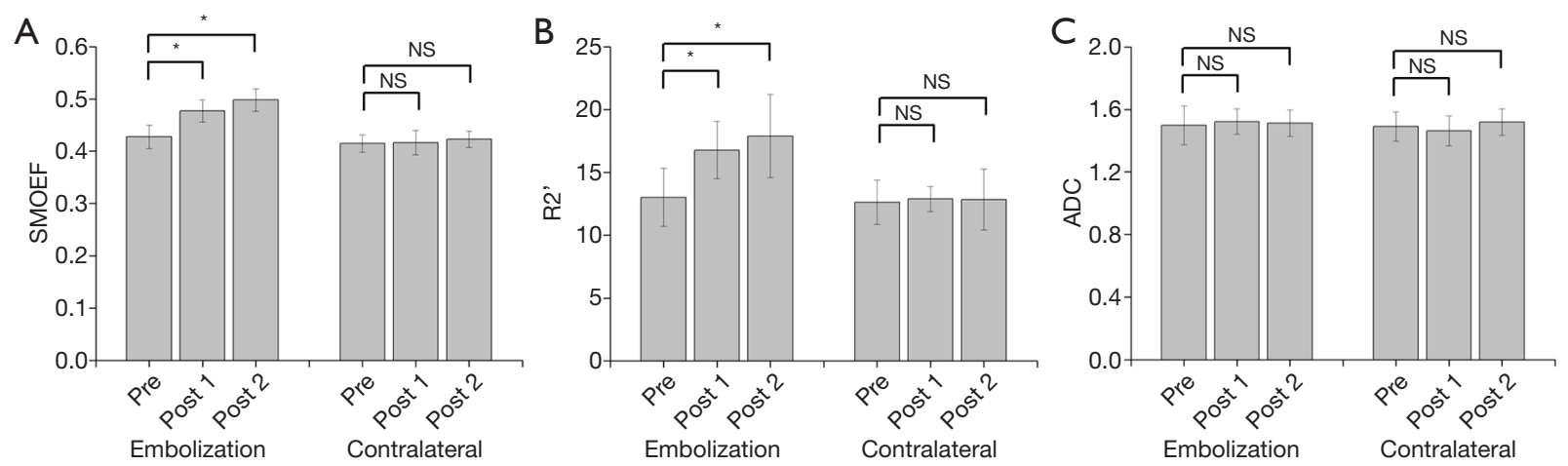

Figure 5 The average SMOEF, R2' and ADC values of all ALI rabbits. (A) The average value of SMOEF of the ischemic leg increases from $0.43 \pm 0.02$ (Pre) to $0.48 \pm 0.02$ (Post $1, \mathrm{P}<0.05$ ), and to $0.50 \pm 0.02$ (Post 2, $\mathrm{P}<0.05$ ); (B) the average R2' increases from 13.01 $\pm 2.31 \mathrm{~s}^{-1}$ (Pre) to $16.78 \pm 2.28 \mathrm{~s}^{-1}$ (Post $1, \mathrm{P}<0.05$ ), and to $17.90 \pm 3.29 \mathrm{~s}^{-1}$ (Post 2, $\left.\mathrm{P}<0.05\right)$; (C) there is no change in average ADC values of the ischemic calf muscle after the embolization procedure [Pre: $(1.50 \pm 0.12) \times 10^{-3} \mathrm{~mm}^{2} / \mathrm{s}$; Post $1:(1.52 \pm 0.08) \times 10^{-3} \mathrm{~mm}^{2} / \mathrm{s}, \mathrm{P}=0.60 ; \mathrm{Post} 2:(1.51 \pm 0.09) \times 10^{-3}$ $\mathrm{mm}^{2} / \mathrm{s}, \mathrm{P}=0.76$ ]. (A,B,C) The average SMOEF, R2' and ADC values of the contralateral legs remain stable after embolization. ${ }^{*} \mathrm{P}<0.05$ with paired Students t-test. ALI, acute limb ischemia; NS, non-significant; SMOEF, skeletal muscle oxygenation extraction fraction; ADC, apparent diffusion coefficient. 
A

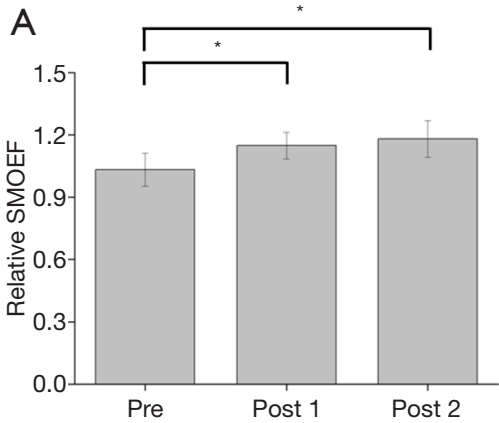

B

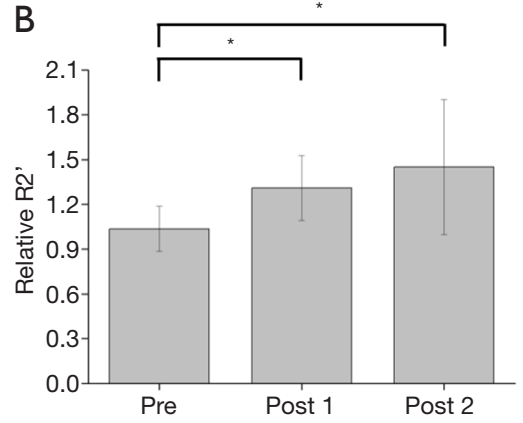

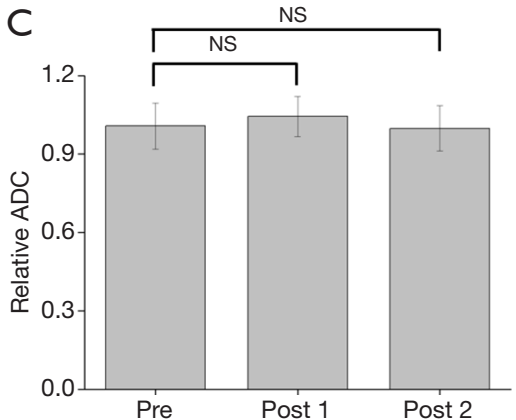

Figure 6 The relative SMOEF, R2' and ADC values of all ALI rabbits. (A) The average relative SMOEF increases from $1.03 \pm 0.08$ (Pre) to $1.15 \pm 0.06$ (Post $1, \mathrm{P}<0.05$ ) and $1.18 \pm 0.09$ (Post 2, $\mathrm{P}<0.05$ ); (B) the average relative R2' increases from $1.04 \pm 0.15$ (Pre) to $1.31 \pm 0.21$ (Post 1 , $\mathrm{P}<0.05$ ) to $1.45 \pm 0.45$ (Post $2, \mathrm{P}<0.05$ ); (A) the relative $\mathrm{ADC}$ values are similar at different stages $(\mathrm{P}=0.33$ and $\mathrm{P}=0.82)$. ${ }^{*} \mathrm{P}<0.05$ with paired Students t-test; ALI, acute limb ischemia; NS, non-significant; SMOEF, skeletal muscle oxygenation extraction fraction; ADC, apparent diffusion coefficient.

the traditional ligation methods (16). This procedure is minimally invasive, and no blood is produced in the region of interest. Since the magnetic susceptibility-based oxygenation measurement is sensitive to changes in oxyhemoglobin and deoxyhemoglobin, the trans-arterial embolization model can avoid the influence of blood at the groin that may be produced by the ligation method. Moreover, different severities of ischemia can be easily induced in this way by adjusting the dose of microspheres in future studies.

There are some limitations to this study. Firstly, several minutes were required for the MEGSE sequence for muscle oxygenation imaging to be conducted. Oxygenation variations might occur during the scanning time, which was ignored in this study. An alternative solution is to use some fast imaging methods (i.e., echo planar imaging) to accelerate the acquisition time. Secondly, calf muscle perfusion was not measured in the current research. This was because most current MRI techniques for muscle perfusion quantification require exogenous contrast agencies like gadolinium diethylenetetramine pentaacetic acid (Gd-DTPA). This could influence the reliability of the susceptibility-based oxygenation imaging technique. Although blood signals have been used as endogenous contrast agents for multi-organ perfusion imaging by the arterial spin labeling technique $(17,18)$, their quality and accuracy are still insufficient for skeletal muscle perfusion imaging.

\section{Conclusions}

We explored the feasibility of using a magnetic resonance susceptibility-based imaging technique to assess muscle oxygenation alternations in ALI models. A significant increase of calf muscle SMOEF was observed in ischemic limbs, which makes the technique a promising tool for noninvasive staging and risk stratification of ALI at an early stage.

\section{Acknowledgments}

Funding: This work was supported by National Natural Science Foundation of China [grant number 81571666] and Peking University First Hospital [grant number 2018SF023, grant number 2018CR16 and grant number 2018CR33].

\section{Footnote}

Conflicts of Interest: The authors have no conflicts of interest to declare.

Ethical Statement: The authors are accountable for all aspects of the work in ensuring that questions related to the accuracy or integrity of any part of the work are appropriately investigated and resolved. The experimental protocols and all embolization procedures were scrutinized by and received approval from the local Institutional Animal Care and Use Committee (J201740).

Open Access Statement: This is an Open Access article distributed in accordance with the Creative Commons Attribution-NonCommercial-NoDerivs 4.0 International License (CC BY-NC-ND 4.0), which permits the noncommercial replication and distribution of the article with 
the strict proviso that no changes or edits are made and the original work is properly cited (including links to both the formal publication through the relevant DOI and the license). See: https://creativecommons.org/licenses/by-nc-nd/4.0/.

\section{References}

1. Aboyans V, Ricco JB, Bartelink MEL, et al. 2017 ESC Guidelines on the Diagnosis and Treatment of Peripheral Arterial Diseases, in collaboration with the European Society for Vascular Surgery (ESVS): Document covering atherosclerotic disease of extracranial carotid and vertebral, mesenteric, renal, upper and lower extremity arteriesEndorsed by: the European Stroke Organization (ESO)The Task Force for the Diagnosis and Treatment of Peripheral Arterial Diseases of the European Society of Cardiology (ESC) and of the European Society for Vascular Surgery (ESVS). Eur Heart J 2018;39:763-816.

2. Creager MA, Kaufman JA, Conte MS. Acute Limb Ischemia. N Engl J Med 2012;366:2198-206.

3. Norgren L, Hiatt WR, Dormandy JA, et al. Inter-Society Consensus for the Management of Peripheral Arterial Disease (TASC II). J Vasc Surg 2007;45 Suppl S:S5-67.

4. Simon F, Oberhuber A, Floros N, et al. Acute Limb Ischemia-Much More Than Just a Lack of Oxygen. Int J Mol Sci 2018;19. doi: 10.3390/ijms19020374.

5. Olinic DM, Stanek A, Tataru DA, et al. Acute Limb Ischemia: An Update on Diagnosis and Management. J Clin Med 2019;8. doi: 10.3390/jcm8081215.

6. Friedrich MG, Karamitsos TD. Oxygenation-sensitive cardiovascular magnetic resonance. J Cardiovasc Magn Reson 2013;15:43.

7. Yang HJ, Oksuz I, Dey D, et al. Accurate needle-free assessment of myocardial oxygenation for ischemic heart disease in canines using magnetic resonance imaging. Sci Transl Med 2019;11. doi: 10.1126/scitranslmed.aat4407.

8. Boezeman RP, Moll FL, Unlu C, et al. Systematic review of clinical applications of monitoring muscle tissue

Cite this article as: Zhang B, Wang C, Wang $\mathrm{H}$, Kong $\mathrm{H}$, Gao F, Yang M, Zhang J. Feasibility of MRI based oxygenation imaging for the assessment of acute limb ischemia. Ann Transl Med 2020;8(6):315. doi: 10.21037/atm.2020.02.139 oxygenation with near-infrared spectroscopy in vascular disease. Microvasc Res 2016;104:11-22.

9. Ledermann HP, Schulte AC, Heidecker HG, et al. Blood oxygenation level-dependent magnetic resonance imaging of the skeletal muscle in patients with peripheral arterial occlusive disease. Circulation 2006;113:2929-35.

10. Yablonskiy DA, Haacke EM. Theory of NMR signal behavior in magnetically inhomogeneous tissues: the static dephasing regime. Magn Reson Med 1994;32:749-63.

11. Zheng J, An H, Coggan AR, et al. Noncontrast skeletal muscle oximetry. Magn Reson Med 2014;71:318-25.

12. Wang $\mathrm{C}$, Zhang $\mathrm{R}$, Zhang $\mathrm{X}$, et al. Noninvasive measurement of lower extremity muscle oxygen extraction fraction under cuff compression paradigm. J Magn Reson Imaging 2016;43:1148-58.

13. An H, Lin W. Quantitative measurements of cerebral blood oxygen saturation using magnetic resonance imaging. J Cereb Blood Flow Metab 2000;20:1225-36.

14. He X, Zhu M, Yablonskiy DA. Validation of oxygen extraction fraction measurement by qBOLD technique. Magn Reson Med 2008;60:882-8.

15. Wang C, Zhang R, Wang R, et al. Noninvasive measurement of renal oxygen extraction fraction under the influence of respiratory challenge. J Magn Reson Imaging 2016;44:230-7.

16. Paek R, Chang DS, Brevetti LS, et al. Correlation of a simple direct measurement of muscle $\mathrm{pO}(2)$ to a clinical ischemia index and histology in a rat model of chronic severe hindlimb ischemia. J Vasc Surg 2002;36:172-9.

17. Qiu D, Straka M, Zun Z, et al. CBF measurements using multidelay pseudocontinuous and velocity-selective arterial spin labeling in patients with long arterial transit delays: comparison with xenon CT CBF. J Magn Reson Imaging 2012;36:110-9.

18. Dang $Y, W u$ B, Sun $Y$, et al. Quantitative assessment of external carotid artery territory supply with modified vessel-encoded arterial spin-labeling. AJNR Am J Neuroradiol 2012;33:1380-6. 